\title{
A furtive stare at an intra-cellular flow
}

\section{T. M. SQUIRES}

Department of Chemical Engineering, University of California, Santa Barbara, CA 93106-5080, USA

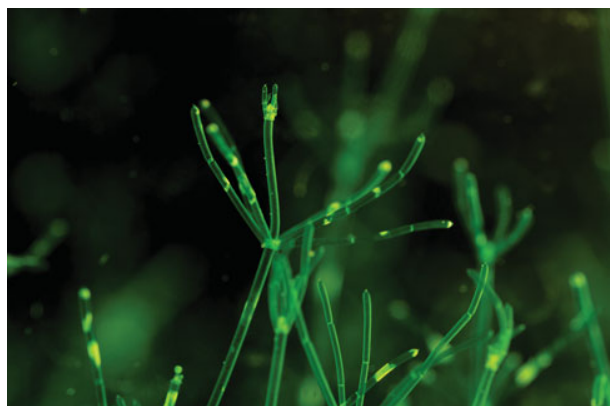

Rarely do intra-cellular flows amount to much: cells are small, and so are their Reynolds numbers. The extraordinarily large cells of the Characean algae provide a fascinating counter-example, as their geometry precludes the standard methods of distributing food and waste. van de Meent et al. (J. Fluid Mech., 2010, this issue, vol. 642 , pp. 5-14) present nuclear magnetic resonance (NMR) velocimetry measurements of the fluid flow within individual living cells, which agree quantitatively with their fluid mechanical model and confirm a long-standing hypothesis. In addition to biomimetic parallels with microfluidic labs on chips, this work showcases NMR velocimetry as an under-appreciated but immensely powerful technique. The non-invasive tracer-free high-resolution flow measurements it enables - even in opaque and heterogeneous fluids - should find wide application.

Key words: biological fluid dynamics, low-Reynolds-number flows

\section{Introduction}

Nature has long inspired researchers in fluid mechanics to explore the mechanical strategies used by living creatures (Vogel 1988). Transport strategies that work at one scale may be ineffective at another (McMahon \& Bonner 1983). The well-known lecture 'Life at Low Reynolds Number' of Purcell (1977) highlights the specific physical demands imposed upon moving micro-organisms (Lauga \& Powers 2009). Life on the interface, too, has succumbed to fluid mechanical analysis: water-walking lizards (Glasheen \& McMahon 1996) achieve their feat very differently from water-striding bugs (Bush \& $\mathrm{Hu}$ 2006). Work on the unsteady hydrodynamics of insect flight (Wang 2005) challenges the apocryphal slur that aerodynamic theory deems bumblebee flight impossible.

In addition to wondering at the world around us - a worthwhile endeavour practical concerns motivate such enquiry. Where better to look for innovative solutions to a technological challenge than to organisms that have had millions of years to devise strategies for related challenges? The drag-reducing qualities of shark skin (Bechert et al. 2000) inspired swimsuits so effective that they have been banned. Synthetic microfluidic 'leaves' may be able to pull clean water from salty sources, as swamp plants do (Wheeler \& Stroock 2008).

However, there has been little attention paid to the fluid mechanics 'within' cells. Cells are generally small enough to quench internal flows, and nutrients, toxins and signals are typically transported either diffusively or through dragging by molecular 
(a)

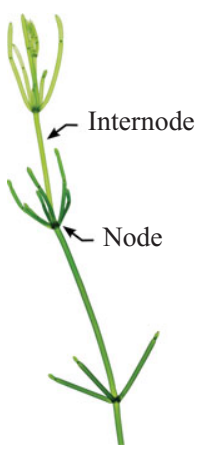

(b)

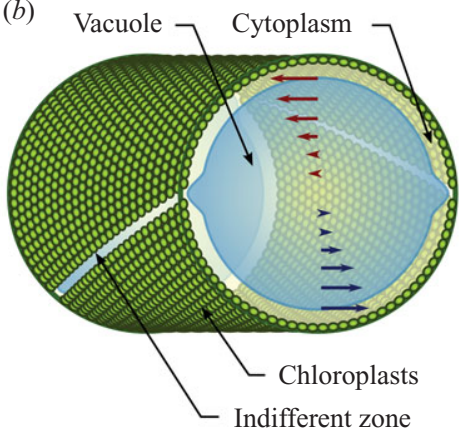

(c)

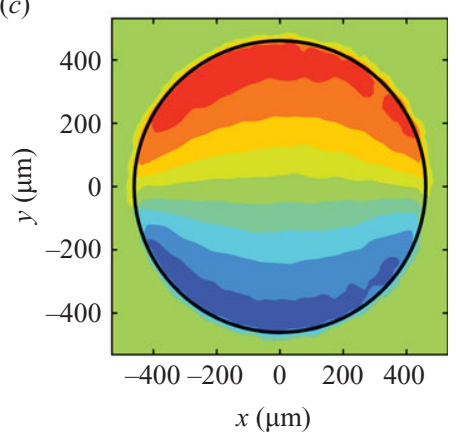

Figure 1. (a) Internode 'stalks' of the Chara Corallina algae consist of single, millimetre-diameter and $\sim 10 \mathrm{~cm}$ long cells. $(b)$ Two helical, counter-directed fluidic "conveyor belts' are established along internode walls, driving the cytoplasm, the vacuolar membrane and its fluid contents into motion. (c) NMR velocimetry profile of the flow within a cell.

motors along filaments. The Characean algae, studied in the present issue by van de Meent et al. (2009), provide a fascinating counter-example. The extraordinarily large internodal cells of these algae face particular challenges: they are small enough for the Reynolds numbers to be low - ruling out turbulent transport or mixing - yet much too large for diffusion to operate on reasonable time scales. To facilitate internal transport and mixing, Characean algae have long been known to employ counter-directed 'conveyor belts' along their walls that drive the fluids within. Previously, Goldstein and co-workers had posed and analysed a model for this transport, whose qualitative features were consistent with available observations (Goldstein, Tuval \& van de Meent 2008; van de Meent, Tuval \& Goldstein 2008). Here, they directly measured the flow within individual living cells - using state-of-the art nuclear magnetic resonance (NMR) techniques and found striking agreement with their predictions.

\section{Overview}

The Characean family of algae contain structures that could be mistaken for the stalks or stems of more conventional plants. However, these 'internodes' - which measure up to $10 \mathrm{~cm}$ in length and $1 \mathrm{~mm}$ in diameter - are single cells (figure $1 \mathrm{a}$ ). Their interior consists almost entirely of a central, membrane-lined vacuole, which serves as a reservoir for food, toxins and wastes while imparting mechanical rigidity to the cell. The vacuole is little more than a bag of fluid and contains no structures that could actively transport its contents (figure $1 b$ ). Clearly, neither the internode cell nor the organism itself could survive if solute transport within the vacuole were purely diffusive: even highly mobile salt ions would require tens of minutes to diffuse across the internode and months to diffuse along it.

These algae solved this transport problem using 'cytoplasmic streaming', which is hypothesized to work as follows. Actin filaments line the inner wall of the internode cell in two oppositely polarized, helical bands. Myosin, a molecular motor, moves along these directed filaments and establishes two oppositely directed 'conveyor belts' that entrain the surrounding cytoplasmic fluid. The shear stress on the vacuolar membrane then drives the internal fluid into motion (figure 1b). This hypothesis 
requires stress to be transmitted from the cytoplasm to the vacuolar fluid, which requires an incompressible, two-dimensional flow within the vacuolar membrane.

Previously, Goldstein's group modelled internodal transport by solving a helical Stokes flow problem in a cylindrical geometry, with effective wall velocities corresponding to motor motion along the polarized filaments (Goldstein et al. 2008; van de Meent et al. 2008). Here, they used NMR velocimetry to make quantitative measurements of the flow profiles within individual internodal cells (figure $1 c$ ). The agreement between the model and the measurements is remarkable.

NMR has seen extensive use for medical imaging and molecular spectroscopy but limited use for velocimetry in the physical sciences. Although its value for rheological studies is becoming increasingly apparent (Callaghan 1999; Bonn et al. 2008), NMR remains largely unfamiliar to the fluid mechanics community. NMR enables non-invasive velocimetry measurements in opaque or heterogeneous fluids without introducing or identifying flow tracers, suggesting far wider application in fluid mechanics. Here, for example, measurements were performed within living cells, which (if cognizant) would have no idea they were being probed.

Briefly, atomic nuclei possess quantized magnetic 'spins', which precess at a precise frequency under an imposed magnetic field. NMR imaging employs carefully designed magnetic fields, gradients and resonant pulses to manipulate and detect this precession in specific regions of space. In NMR velocimetry, a spin pattern is 'written' into a region of interest, and its evolution because of flow is detected. Dedicated practitioners can achieve $\sim 10 \mu \mathrm{m}$ resolution velocity profiles in systems that may otherwise be completely inaccessible.

\section{Future}

From a pure science standpoint, the hypothesis behind cytoplasmic streaming - and the fluid mechanical model employed for its description - have been directly confirmed. On the biomimetic front, these algae face the same conundrum as microfluidic 'labs on chips', where fluids must be transported and mixed within channels that are tens to hundreds of microns in height and centimetres in length (Stone, Stroock \& Ajdari 2004; Squires \& Quake 2005). The well-known microfluidic mixer of Stroock et al. (2002) modulates the fluid boundary conditions on channel walls to drive stirring flows, much like the Characean algae. This should serve as a potent reminder that the immense variety of organisms on Earth contains a wealth of expertise that may be mined for biomimetic solutions.

More broadly, the NMR velocimetry techniques used to make these measurements are incredibly powerful and merit more widespread use in fluid mechanics. The technique is completely non-invasive, requires no flow tracers and can be performed in non-transparent materials. NMR has revealed inhomogeneous (shear-banding) flows in yield-stress materials (Bonn et al. 2008). Degre et al. (2006) extracted the shearrate-dependent viscosities of complex fluids from pressure-driven velocity profiles in microchannels, which they measured using micro-particle image velocimetry. NMR velocimetry should enable similar measurements for a much broader range of materials, complementing other techniques for microfluidic viscometry (Pipe \& McKinley 2009). While microfluidic systems are usually viewed as projections 'from above', NMR can capture velocity profiles 'across' microchannels. It would also be capable of measuring cross-sectional concentration fields of selected chemical species, e.g. without requiring fluorescent labels. Such information, obtained using 
three-dimensional fluorescence confocal microscopy (Ismagilov et al. 2000), played a key role in the development of the T-sensor.

It would strike most physicists as ironic that the fluid mechanics community knows Purcell primarily as the author of an elegant and thought-provoking lecture on the low-Reynolds world of the micro-organism. Few among us are aware of his primary claim to scientific fame: his development of solution- and solid-state NMR, for which he was awarded the Nobel Prize in 1952. Given that it was Purcell's lecture that inspired many of us to see micro-organisms in a new way, it seems particularly fitting to find one of their mysteries unlocked by Purcell's own technique.

\section{References}

Bechert, D. W., Bruse, M., Hage, W. \& Meyer, R. 2000 Fluid mechanics of biological surfaces and their technological application. Naturwissenschaften 87 (4), 157-171.

Bush, J. W. M. \& Hu, D. L. 2006 Walking on water: biolocomotion at the interface. Annu. Rev. Fluid Mech. 38, 339-369.

Bonn, D., Rodts, S., Groenink, M., Rafai, S., Shahidzadeh-Bonn, N. \& Coussot, P. 2006 Some applications of magnetic resonance imaging in fluid mechanics: complex flows and complex fluids. Annu. Rev. Fluid Mech. 40, 209-233.

Callaghan, P. T. 1999 Rheo-NMR: nuclear magnetic resonance and the rheology of complex fluids. Rep. Progr. Phys. 62 (4), 599-670.

Degre, G., Joseph, P., Tabeling, P., Lerouge, S., Cloitre, M. \& Ajdari, A. 2006 Rheology of complex fluids by particle image velocimetry in microchannels. Appl. Phys. Lett. 89 (2), 024104.

Glasheen, J. W. \& McMahon, T. A. 1996 A hydrodynamic model of locomotion in the basilisk lizard. Nature 380 (6572), 340-342.

Goldstein, R. E., Tuval, I. \& van De Meent, J. W. 2008 Microfluidics of cytoplasmic streaming and its implications for intracellular transport. Proc. Natl Acad. Sci. USA 105 (10), 3663-3667.

Ismagilov, R. F., Stroock, A. D., Kenis, P. J. A., Whitesides, G. \& Stone, H. A. 2000 Experimental and theoretical scaling laws for transverse diffusive broadening in two-phase laminar flows in microchannels. Appl. Phys. Lett. 76, 2376-2378.

Lauga, E. \& Powers, T. R. 2009 The hydrodynamics of swimming microorganisms. Rep. Progr. Phys. 72 (9), 096601.

McMahon, T. A. \& Bonner, J. T. 1983 On Size and Life. Scientific American Library.

van de Meent, J. W., Sederman, A. J., Gladden, L. F. \& Goldstein, R. E. 2010 Measurement of cytoplasmic streaming in single plant cells by magnetic resonance velocimetry. J. Fluid Mech. 642, 5-14.

van de Meent, J. W., Tuval, I. \& Goldstein, R. E. 2008 Nature's microfluidic transporter: rotational cytoplasmic streaming at high Péclet numbers. Phys. Rev. Lett. 101 (17), 178102.

PiPe, C. J. \& McKinley, G. H. 2009 Microfluidic rheometry. Mech. Res. Commun. 36 (1), 110-120.

Purcell, E. M. 1977 Life at low Reynolds number. Am. J. Phys. 45 (1), 3-11.

SQuires, T. M. \& QuaKe, S. R. 2005 Microfluidics: fluid physics at the nanoliter scale. Rev. Mod. Phys. 77 (3), 977-1026.

Stone, H. A., Stroock, A. D. \& AJdARI, A. 2004 Engineering flows in small devices: microfluidics toward a lab-on-a-chip. Annu. Rev. Fluid Mech. 36, 381411.

Stroock, A. D., Dertinger, S. K. W., Ajdari, A., Mezic, I., Stone, H. A. \& Whitesides, G. M. 2002 Chaotic mixer for microchannels. Science 295 (5555), 647-651.

Vogel, S. 1988 Life's Devices. Princeton University Press.

WANG, Z. J. 2005 Dissecting insect flight. Annu. Rev. Fluid Mech. 37, 183-210.

Wheeler, T. D. \& Stroock, A. D. 2008 The transpiration of water at negative pressures in a synthetic tree. Nature 455 (7210), 208-212. 